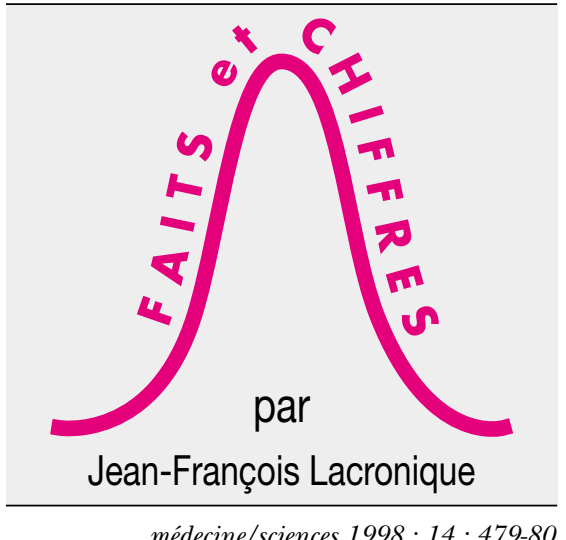

médecine/sciences 1998 ; 14 : 479-80

\section{Démographie médicale}

On a recensé, en 1995, 650000 médecins actifs aux États-Unis. Un tiers peut être classé en «médecine générale » (ou de soins primaires), tandis que les deux tiers sont spécialistes.

On ajoute habituellement à ces chiffres certains praticiens de médecine ostéopathique, au nombre de 35000 , dont la moitié se range en « soins primaires".

Le rythme de croissance du nombre des médecins est plus rapide que celui de la population générale et, de ce fait, le rapport médecins/ population est croissant, atteignant en 1995 le chiffre de 280/100 000, soit plus élevé de $29 \%$ qu'en 1980 (Tableau I).

L'inertie du système est telle que l'on sait aujourd'hui que le nombre de médecins continuera de croître irréversiblement, à cause de l'attrait des hôpitaux américains pour les médecins d'origine étrangère. Ceuxci représentent $22,7 \%$ des médecins praticiens aux États-Unis.

La plupart des analystes s'accordent pour dire que la démographie médicale aux Etats-Unis est largement excédentaire, depuis environ quinze ans. Pourtant, il se crée encore de nouvelles écoles de médecine (la dernière, une école de chiropracteurs, s'est ouverte en 1993).

Cependant, cet excédent de médecins ne permet pas de résorber les déficits qui existent encore aux États-Unis, notamment dans des

Source : Physician characteristics and distribution in the US, AMA, 1996.

\title{
Quelques chiffres concernant la médecine aux États-Unis
}

\begin{tabular}{|c|c|c|}
\hline \multicolumn{3}{|c|}{ LES MÉDECINS AMÉRICAINS, PAR TYPE D'ACTIVITÉ } \\
\hline Types d'activité & 1985 & 1994 \\
\hline Soins aux malades & 448820 & 543200 \\
\hline Cabinet & 330197 & 407000 \\
\hline Hôpital & 118623 & 136100 \\
\hline résidents & 75411 & 86800 \\
\hline Autres activités & 48320 & 42888 \\
\hline enseignement & 7832 & 7983 \\
\hline administration & 13810 & 14923 \\
\hline recherche & 23268 & 16367 \\
\hline Total & 552716 & 684400 \\
\hline
\end{tabular}

\begin{tabular}{|c|c|c|c|c|}
\hline \multicolumn{5}{|c|}{ Tableau II } \\
\hline & \multicolumn{2}{|c|}{ Total en milliards $\$$} & \multicolumn{2}{|c|}{ Par personne } \\
\hline Années & \$ courant & \$ constant & \$ courant & $\$$ constant \\
\hline 1980 & 251 & 452 & 1068 & 1926 \\
\hline 1985 & 434 & 599 & 1761 & 2428 \\
\hline 1990 & 696 & 790 & 2684 & 3048 \\
\hline 1991 & 755 & 822 & 2880 & 3136 \\
\hline 1992 & 820 & 867 & 3094 & 3271 \\
\hline 1993 & 884 & 907 & 3301 & 3388 \\
\hline 1994 & 940 & 940 & 3478 & 3478 \\
\hline 1995 & 1007 & 977 & 3690 & 3579 \\
\hline
\end{tabular}

zones rurales ou sous-développées. La croissance la plus forte se trouve dans les zones déjà les plus médicalisées, notamment dans les grandes villes de la côte est, en Floride et en Californie. Enfin, malgré tous les efforts engagés dans le sens d'un rééquilibrage du rapport généralistes/spécialistes, la médecine de soins primaires n'attire qu'un nombre très insuffisant de jeunes médecins: le nombre d'internes se dirigeant vers cette filière est tombé en 1992 à 14,6 \%.

Plus spécifique aux États-Unis, la question d'un équilibrage entre les différentes ethnies montre que les minorités sont mal représentées en médecine. Seule la féminisation de la profession a marqué un progrès, le nombre des femmes s'étant accru de $35 \%$ entre 1981 et 1990 . Mais le 
nombre des médecins noirs a diminué de $3,1 \%$ à $3 \%$ pendant la même période, et celui des hispaniques est passé de $4,4 \%$ à $4,5 \%$ (alors que la population de même origine est passé de 13 à 25 millions).
Dépenses de santé : données synthétiques

Les 270 millions d'Américains ont consommé pour leur santé plus de mille milliards de dollars en 1995 :

\begin{tabular}{|c|c|c|c|c|}
\hline \multicolumn{5}{|c|}{$\begin{array}{l}\text { Tableau III } \\
\text { DE SANTÉ AUX ÉTATS-UNIS, } \\
\text { RCE DE FINANCEMENT }\end{array}$} \\
\hline $\begin{array}{l}\text { Source } \\
\text { du financement } \\
\text { (en milliards de \$) }\end{array}$ & 1980 & 1985 & 1990 & 1995 \\
\hline Medicare & 37,5 & 72,2 & 112,1 & 187,8 \\
\hline Medicaid & 26,1 & 41,3 & 75,4 & 138,4 \\
\hline Autre public & 41,6 & 61,6 & 99 & 128 \\
\hline Privé & 145,8 & 259,4 & 410 & 533,8 \\
\hline Assurance & 72,1 & 139,8 & 237 & 306 \\
\hline Ménage & 61,3 & 98,8 & 138,3 & 187,8 \\
\hline Autre privé & 12,4 & 20,8 & 34,3 & 39,5 \\
\hline Total & 251,1 & 434,5 & 696,6 & 988,5 \\
\hline
\end{tabular}

\begin{tabular}{|lcccc|}
\hline \multicolumn{4}{c}{ Tableau IV } \\
& SOURCE DU FINANCEMENT, EN POURCENTAGE \\
\hline $\begin{array}{l}\text { Source } \\
\text { du financement } \\
\text { (en \%) }\end{array}$ & 1980 & 1985 & 1990 & 1995 \\
\hline Medicare & & & & \\
Medicaid & 15 & 16,6 & 16,1 & 19 \\
Autre public & 10,4 & 9,5 & 10,8 & 14 \\
Privé & 16,6 & 14,2 & 14,2 & 13 \\
$\begin{array}{l}\text { Assurance } \\
\text { Ménage }\end{array}$ & 58,1 & 59,7 & 58,9 & 54 \\
Autre privé & 28,7 & 32,2 & 34 & 31 \\
& 24,4 & 22,7 & 49,9 & 19 \\
\hline
\end{tabular}

Tableau V

LES DÉPENSES DE «SANTÉ PUBLIQUE »

\section{$\mathrm{NIH}$}

Health Resources and Services Administration

Substance Abuse and Mental Health

CDC

FDA

AHCPR

Toxic Substances and Disease Registry

Office of Assistant Secretary for Health
11600

3100

2225

2200

883

148

68

50

$N I H$ : national institute of health ; $C D C$ : center for disease control ; FDA : food and drug adminis-

(1 050 milliards de \$), soit environ 3560 \$ par personne en moyenne (Tableau II).

- L'effort budgétaire en faveur de la santé représente environ $14 \%$ du PIB.

- L'année 1995 confirme la décélération observée depuis 3 ans.

- Le rythme de croissance des dépenses de santé 1995/1994 est de l'ordre de 5,1\%.

La dépense publique est de 44,3\% de la dépense totale (43\% en 1993) (Tableaux III et $I V$ ).

Les deux programmes publics Medicaid et Medicare (le premier couvrant les dépenses de santé des personnes pauvres, le dernier couvrant les personnes âgées de plus de 65 ans) représentent 330 milliards de $\$$ de dépenses, c'est-à-dire $30 \%$ du total, et $70 \%$ de la dépense publique de santé. Les organismes de couverture privés (assurances et mutuelles) ont en charge $57 \%$ de la dépense totale.

Les dépenses qui restent à la charge des usagers représentent une fraction déclinante de la dépense totale, passant de $20 \%$ en 1990 à $19 \%$ en 1995. - Les hôpitaux assurent $37 \%$ de la dépense totale, les médecins $19 \%$.

- La croissance des dépenses s'est beaucoup ralentie entre 1992 et 1995 .

- Les soins hospitaliers sont passés d'un rythme de croissance annuel de $10,7 \%$ à $4,4 \%$.

- Les soins de médecins de $10,3 \%$ à $4,6 \%$.

- Les médicaments prescrits de $14,5 \%$ à $4,5 \%$.

- Le nombre de personnes qui ne bénéficient d'aucune couverture sociale continue de croître, notamment chez les personnes âgées (18\% d'entre elles en sont totalement dépourvues, contre $17 \%$ en 1991) et dans les minorités ethniques.

L'effort budgétaire en "santé publique » (incluant la recherche médicale) en 1996 (en millions de dollars), malgré un Congrès plutôt méfiant à l'égard de toutes dépenses publiques, reste maintenu à un niveau élevé (Tableau V). En 1998, le budget du NIH atteindra 16 milliards de dollars, traduisant un rythme de croissance sans précédent

\section{Jean-François Lacronique}

Office de protection contre les rayonnements ionisants, 31, rue de l'Écluse, BP 35, 78116 le Vésinet Cedex, France. 